\title{
Rate Adaptation Mechanism with Available Data Rate Trimming and Data Rate Information Provision for V2I Communications
}

\author{
Shigeru Kashihara $\mathbb{D D}^{1}{ }^{1}$ Takemi Sahara, ${ }^{1}$ Shigeru Kaneda, ${ }^{2}$ and Chikara Ohta ${ }^{3}{ }^{3}$ \\ ${ }^{1}$ Nara Institute of Science and Technology, Ikoma, Nara 6300192, Japan \\ ${ }^{2}$ Space-Time Engineering, LLC, Rolling Hills Estates, CA, USA \\ ${ }^{3}$ Kobe University, Kobe, Hyogo 6578501, Japan \\ Correspondence should be addressed to Shigeru Kashihara; shigeru@is.naist.jp
}

Received 5 October 2018; Accepted 26 March 2019; Published 15 April 2019

Guest Editor: Mohamed Elhoseny

Copyright (c) 2019 Shigeru Kashihara et al. This is an open access article distributed under the Creative Commons Attribution License, which permits unrestricted use, distribution, and reproduction in any medium, provided the original work is properly cited.

\begin{abstract}
We study a rate adaptation mechanism for improving communication performance between a connected vehicle and a roadside unit (RSU) using Wi-Fi during movement in a vehicle-to-infrastructure (V2I) environment. Wi-Fi communication provides various attractive services to connected vehicles during movement. However, as a connected vehicle is generally moving at high speed, the communication performance with an RSU that works as an access point is degraded because wireless link quality fluctuates abruptly and continuously. We then propose a rate adaptation mechanism employing the following two main features to mitigate such deterioration in communication performance: available data rate trimming and data rate information provision. To alleviate degradation of communication, the former avoids usage of excessively low data rates and the latter then provides data rate information suitable for channel quality from a dataset of adequate data rates based on the vehicle's location and speed. However, the data rate information provided from a dataset may not always be appropriate because of various indefinite factors such as multipath fading and shadowing. Thus, the proposed method also employs a measurement-based function to compensate for such a drawback of the dataset. Simulation experiments evaluate communication performance for 10,60 , and $100 \mathrm{~km} / \mathrm{h}$ in single-vehicle and multiplevehicles cases. Simulation results showed that the proposed method overall provides superior communication performance in situations involving more than one vehicle, in comparison with existing counter- and sample-based methods.
\end{abstract}

\section{Introduction}

With the goal of providing attractive services such as safety information, traffic efficiency management, and entertainment services for connected vehicles, intelligent transport systems (ITSs) are being developed at a fast pace [1]. A network component that connects a vehicle with other vehicles and with intelligent road infrastructures is essential for realizing an ITS. At present, vehicles have communication equipment, and advanced multimedia and infotainment services are about to start via the Internet [2].

As depicted in Figure 1, introducing edge computing [3] to vehicular networks is essential for providing attractive services such as data offloading [4] to connected vehicles. A connected vehicle creates data of various types and sizes and sends them to receive the services from an edge node or a server during movement. After receiving data, the edge node or server then provides appropriate services to the connected vehicle based on the situation analyzed from the data received. To provide such services, vehicle-to-infrastructure (V2I) communication is necessary for gathering a variety of information like the vehicle's position, speed, points of origin and destination, image data, and so on.

In V2I, the IEEE 802.11 series [5] is assumed to be employed for the communication media [6]. However, as a connected vehicle is moving at high speed, it may not have enough time to communicate with a roadside unit (RSU) that works as an access point (AP), because the coverage of an $\mathrm{AP}$ is relatively small. Also, in a high-mobility situation, it 


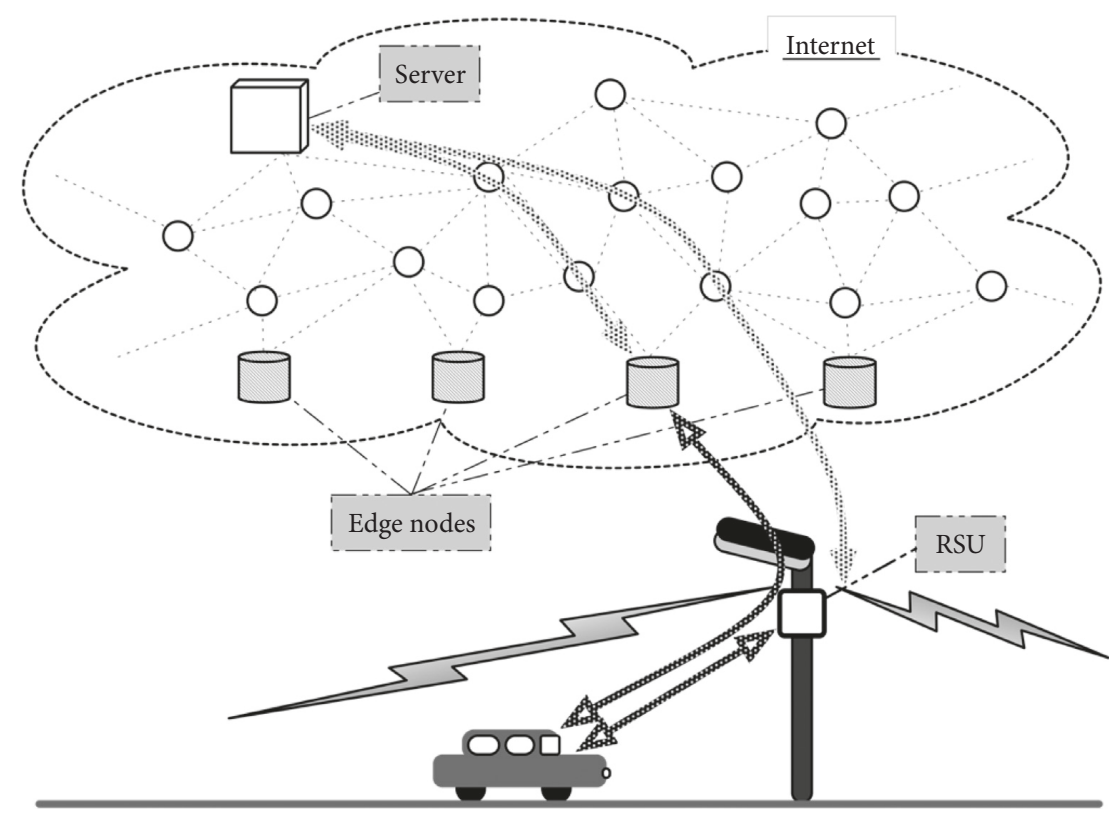

FIgURE 1: Communication between a connected vehicle and an RSU.

is difficult to enhance throughput due to sudden and continuous fluctuations in wireless channel quality. Therefore, improving the communication performance between a connected vehicle and an RSU is essential for providing various services.

In the above situation, as the connected vehicle communicates with single-hop RSUs or small-hops edge nodes, not with a remote server, it is essential to improve communication performance between a connected vehicle and an RSU. This paper, therefore, focuses on a rate adaptation mechanism as one approach to improving such communication performance in a high-mobility situation. Although a rate adaptation mechanism aims to set an appropriate data rate for wireless link conditions during movement, IEEE 802.11 [5] has not standardized a rate adaptation mechanism, and the mechanism, therefore, depends on implementation matters. Moreover, Wi-Fi communication generally employs multiple data rates (e.g., eight types of data rate on IEEE 802.11p) to provide better throughput for changes of channel quality, and each data rate is adjusted by channel quality measurement. However, as Wi-Fi communication was not designed to be employed in a highmobility situation, it is difficult to follow rapidly fluctuating channel conditions at short intervals.

In this paper, to select an appropriate data rate for changes of channel quality, we propose a rate adaptation mechanism that has the following new two features: available data rate trimming and data rate information provision. Available data rate trimming alleviates the degradation of communication performance by reducing usage of excessively low data rates because a low data rate consumes more communication time than a high data rate. Also, the provision of data rate information from a dataset supplies adequate data rates for a vehicle's locations, without any measurement. However, the dataset may include erroneous data rate information for the situation. If the data rate provided from the dataset is not acceptable, the proposed method also utilizes a measurementbased function to mitigate the effects of such a problem. In summary, the significant contributions of our work to improve V2I communication performance in a high-mobility environment are as follows:

(i) Employing higher data rates by trimming regular data rates

(ii) Providing adequate data rate information from a dataset based on a vehicle's information

(iii) Cooperating dataset- and measurement-based functions

We also evaluate the communication performance of the proposed method through simulation experiments to show the effectiveness of the proposed method.

The remainder of the paper is organized as follows: Section 2 surveys existing research work on rate adaptation mechanisms. Section 3 discusses the communication performance of the existing rate adaptation mechanisms. Section 4 presents our proposed method, and Section 5 shows the results of the performance evaluation via simulations. Section 6 provides concluding remarks.

\section{Related Work}

As described in Section 1, rate adaptation mechanism has not been the objective in the standardization of IEEE 802.11 [5]. However, as a rate adaptation mechanism strongly influences communication performance over a wireless link, various rate adaptation mechanisms have been proposed to date [7-23]. In particular, communication performance is strictly dependent on the way channel quality is estimated. Thus, this section first classifies the mechanisms into counter-based and samplebased mechanisms, and we then consider other methods in a vehicular ad-hoc network (VANET) field for comparison. 
Auto rate fallback (ARF) [7] and adaptive ARF (AARF) [8] are representative rate adaptation methods, and they are generally classified as counter-based mechanisms. ARF is the most straightforward mechanism, selecting the best data rate based on the numbers of continuous successful and failed transmissions. Concretely, when a sender successfully transmits a data frame ten consecutive times, it raises the current transmission rate to the next higher transmission rate. Conversely, when data frame transmission fails twice in succession, the current transmission rate is dropped to the next lower transmission rate. To achieve this, the sender counts the number of successful and failed data frame transmissions to estimate channel quality and changes the transmission rate when the count reaches the predetermined threshold.

Onoe [16, 17], SampleRate [16], and Minstrel [17, 18] are representative sample-based mechanisms. In the samplebased mechanism, to estimate channel quality, a sender employs statistical information such as retransmission rate, packet error rate, and throughput based on a sliding window process. For instance, in Onoe, the sender measures the number of data frame retransmissions within a window time (e.g., one second) and then compares the measurement result with a predetermined rate. If the result is $10 \%$ or less, a credit counter is increased by one. On the one hand, the credit counter is decreased by one if the result is $10 \%$ or more. When the credit counter reaches ten points, the sender moves the transmission rate up to the next higher one. If the sender experiences failed data frame transmission of over $50 \%$, the transmission rate is moved down to the next lower one.

Also, in [19-21] a signal-to-noise ratio (SNR) is utilized, but it is difficult to obtain accurate SNR values in a practical environment. In [13] a threshold optimization algorithm for a rate adaptation mechanism that employs up/down threshold is proposed, and in [14] a rate adaptation algorithm that uses short-term loss ratio and an adaptive RTS filter is designed and implemented. The above approaches provide good communication performance for an environment where a sender and a receiver are stationary. However, if the sender moves at high speed like a vehicle, it is difficult to estimate changes of channel quality and set an appropriate transmission data rate based on the measurement result, because the measured channel quality may be outdated for the channel quality at the present location.

We describe rate adaptation methods focusing on a vehicular ad-hoc network (VANET). In [22] database for providing information of adequate data rate based on information of vehicle's location, i.e., location, velocity, and density, similar to with one of our approaches, is employed, but it may be difficult to obtain the density information of surrounding vehicles. The studies [9-12, 23] need parameter adjustments beforehand for particular environments, but such prior adjustments are difficult to apply in various situations. Besides, in [24] existing rate adaptation algorithms in vehicular networks with IEEE802.11p are evaluated, but it shows only that the communication performance of constant bit rate (CBR) for them via ns-3 simulations. As ITS including edge computing provides multiple services such as safety information, traffic efficiency management, and entertainment services to a connected vehicle, it is significant to improve the communication performance between a connected vehicle and an RSU. In this study, we, therefore, investigate a rate adaptation method that does not require such prior adjustments and consider the communication performance of UDP and TCP traffics for multiple services provided.

\section{Communication Performance of Existing Rate Adaptation Mechanisms}

This section presents the communication performance of existing rate adaptation mechanisms through simulation experiments. We use ARF and Onoe as representative counter-based and sample-based mechanisms, respectively, because they have been widely deployed in products. Section 3.1 describes the simulation model and parameters. Section 3.2 shows communication performance of file transfer protocol (FTP) and CBR applications for each mechanism at three speeds, i.e., 10,60 , and $100 \mathrm{~km} / \mathrm{h}$.

3.1. Simulation Model and Parameters. As depicted in Figure 2, in the simulation model, a connected vehicle passes by an RSU, an AP of a wireless communication infrastructure, in a straight line at three constant speeds of 10,60 , and $100 \mathrm{~km} / \mathrm{h}$. The simulation model also employs FTP and CBR applications to evaluate communication performance. The FTP application tries to send 2048 MByte data from the vehicle to the RSU, while the CBR application tries to send one 1500-byte packet per $222 \mu$ s from the vehicle to the RSU. The RSU is assumed to employ IEEE $802.11 \mathrm{p}$, which provides eight data rates $(3,4,5,6,9,12,18,24$, and $27 \mathrm{Mbit} / \mathrm{s})$. The transmission powers of the RSU and the vehicle are set to $20 \mathrm{dBm}$, and the two-ray ground reflection model and Nakagami-m fading model are employed as propagation models. Table 1 summarizes the above parameters. Note that the simulation experiments work on Scenargie ${ }^{\circledR}$ Simulator (Space-Time Engineering, LLC, "Scenargie ${ }^{\circledR}$ Simulator," https://www.spacetime-eng.com/en/products), a commercial product for analyzing and evaluating wireless communications and networking systems.

3.2. Communication Performance during Movement. The simulation experiments investigate how much communication performance the existing rate adaptation mechanisms can obtain at the three movement speeds. Figure 3 shows the results of FTP and CBR applications for ARF and Onoe at the speed of $60 \mathrm{~km} / \mathrm{h}$, as an example. In these simulation results, the best value (Best) means the largest amount of data that the receiver could receive as data packets per $100 \mathrm{~ms}$ among 100 simulation experiments for each transmission rate. This is calculated as follows: we first have 100 simulation trials for eight data rates using the same model. The best performance per $100 \mathrm{~ms}$ is then extracted from 800 simulation results, that is, the best value is constructed from the best performance. In the results of ARF and Onoe, we employ the median value for 100 simulation experiments. 


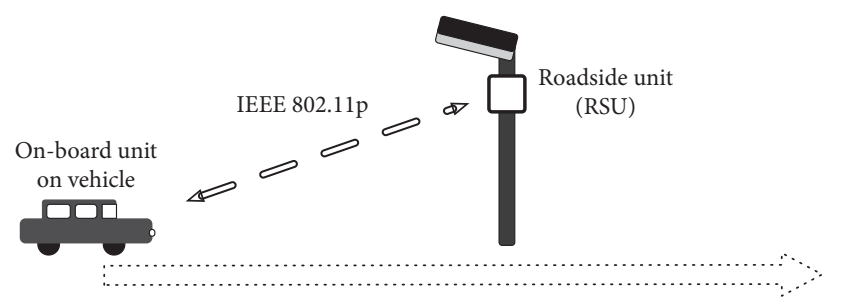

FIGURE 2: Simulation model.

TABLE 1: Simulation parameters.

\begin{tabular}{|c|c|}
\hline Vehicle's speed & $10,60,100 \mathrm{~km} / \mathrm{h}$ \\
\hline Traffic model & $\begin{array}{c}\text { FTP: sends a } 2048-\text { MByte file } \\
\text { CBR: sends a } 1500 \text {-byte packet at } \\
\text { intervals of } 222 \mu \mathrm{s}\end{array}$ \\
\hline Wireless medium & $\begin{array}{c}\text { IEEE } 802.11 \mathrm{p} \text { (data rate: } 3,4.5,6,9,12 \text {, } \\
18,24 \text {, and } 27 \mathrm{Mbit} / \mathrm{s} \text { ) }\end{array}$ \\
\hline $\begin{array}{l}\text { Rate adaptation } \\
\text { mechanism }\end{array}$ & $\begin{array}{cc}\text { ARF (counter- } & \text { Onoe (sample- } \\
\text { based) } & \text { based) }\end{array}$ \\
\hline Transmission power & $20 \mathrm{dBm}$ \\
\hline Propagation model & $\begin{array}{l}\text { Two-ray ground reflection model } \\
\text { Nakagami-m fading model }\end{array}$ \\
\hline $\begin{array}{l}\text { Number of simulation } \\
\text { trials }\end{array}$ & 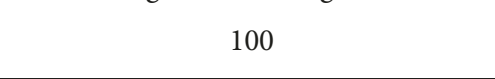 \\
\hline
\end{tabular}

Compared with the best value, we can see that ARF and Onoe cannot achieve a sufficient data rate at the beginning and the end of the communication. From these results, we infer that $\mathrm{ARF}$ is excessively downgrading the data rate used. As described in Section 2, the threshold in ARF for downgrading is very low, i.e., two consecutive failures, but that for upgrading is relatively high, i.e., ten consecutive successes. It can therefore be said that ARF sensitively reacts to lost frames. On the other hand, in Onoe, as the number of data frame retransmissions per second must be measured to estimate channel quality, the measurement period makes suitable channel estimation difficult.

From the above comparison between ARF and Onoe, we can say that ARF obtains the better performance when the vehicle is near the RSU, while Onoe cannot raise the performance to a higher level even if the vehicle is near the AP. This is because, since the change in data rate in ARF is based on the success or failure of frame transmission, ARF can change the data rate relatively fast. However, it does not always follow channel quality. On the other hand, since the changes in data rate in Onoe depend on measurement, Onoe needs more time to decide a change of data rate than ARF. Thus, it is difficult to make use of higher data rates by the conservative change policy of the rate adaptation mechanisms.

Table 2 presents the simulation results of FTP and CBR communications at three speeds of 10,60 , and $100 \mathrm{~km} / \mathrm{h}$. Note that room for improvement means the proportion of actual data received for the best value. From the results, we can see that although ARF provides better performance than Onoe for a moving vehicle, both mechanisms have more room for improvement for higher speed.

\section{Rate Adaptation Mechanism}

The previous section showed that the existing rate adaptation mechanisms have room for improvement in communication performance during movement. We now propose a rate adaptation mechanism employing the following two features to improve the performance: trimming the number of available data rates to four and using a dataset to provide data rate information based on a vehicle's location and speed. Section 4.1 outlines the design overview of our proposed method. Sections 4.2 and 4.3 then explain the above two features of the proposed rate adaptation mechanism. Sections 4.4 and 4.5 additionally describe two change policies for transmission data rate, with and without using information from the dataset.

4.1. Design Overview. As ARF and Onoe adapt the data rate depending only on past measurement information, it is difficult to use an appropriate data rate for the present channel quality during movement at high speed, that is, the measurement information for these existing methods may be stale when current channel quality is being estimated, especially at high speed. Besides, it is almost impossible to make an accurate estimation because the channel quality is changing continuously due to various indeterminate factors. To choose a data rate that is as appropriate as possible for the present channel quality, our proposed method therefore assumes the use of a dataset that provides data rate information based on a vehicle's speed and location.

Under this assumption, the dataset on an RSU collects three-tuple information of the vehicle's speed, location, and data rate used, and it determines an appropriate data rate based on the information collected. As a vehicle obtains RSU information via the Internet beforehand or through a local dynamic map (LDM) service [25], it can choose an appropriate data rate based on the dataset. However, as details of designing and analyzing the dataset constitute another research topic, they are beyond the scope of the present paper. Therefore, we assume here that the dataset has been created from measurement data collected in advance.

Figure 4 depicts the design overview of the proposed method. The existing rate adaptation mechanisms choose a transmission data rate based only on information that can be obtained within the MAC layer. On the other hand, as the proposed method utilizes a vehicle's speed and location information for selecting the data rate, the MAC layer needs to obtain context information (CI) from the Application layer. To access the CI on the Application layer from the MAC layer, a shared memory is employed. In this approach, the Application layer writes CI to the shared memory, while the MAC layer reads the CI from the shared memory and obtains an appropriate data rate based on the CI from the dataset.

However, it is too difficult to make a complete dataset because channel quality is affected by various invisible factors. In the proposed method, when a data rate selected based on the dataset is not appropriate for the channel 


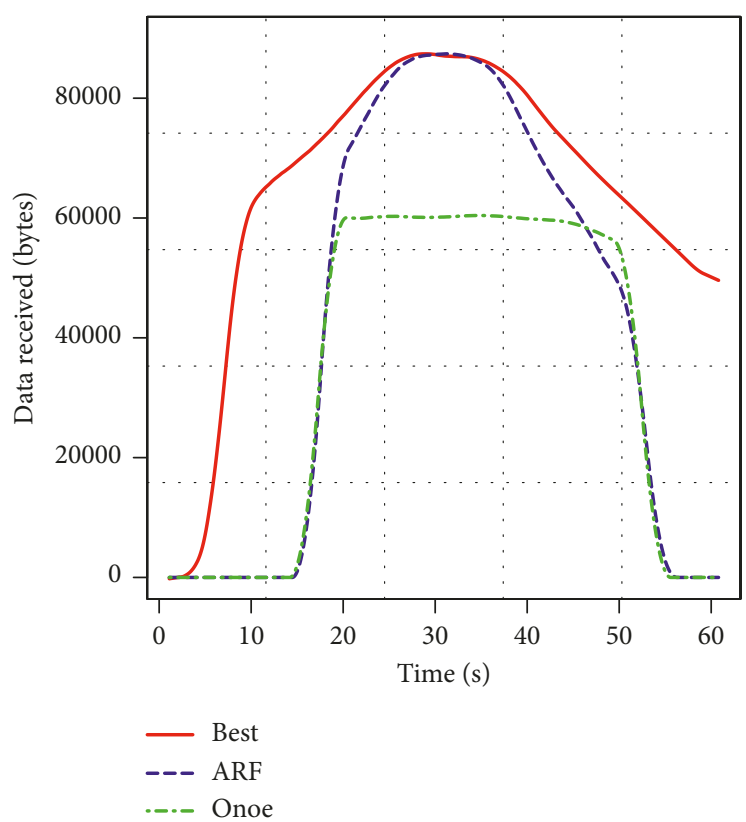

(a)

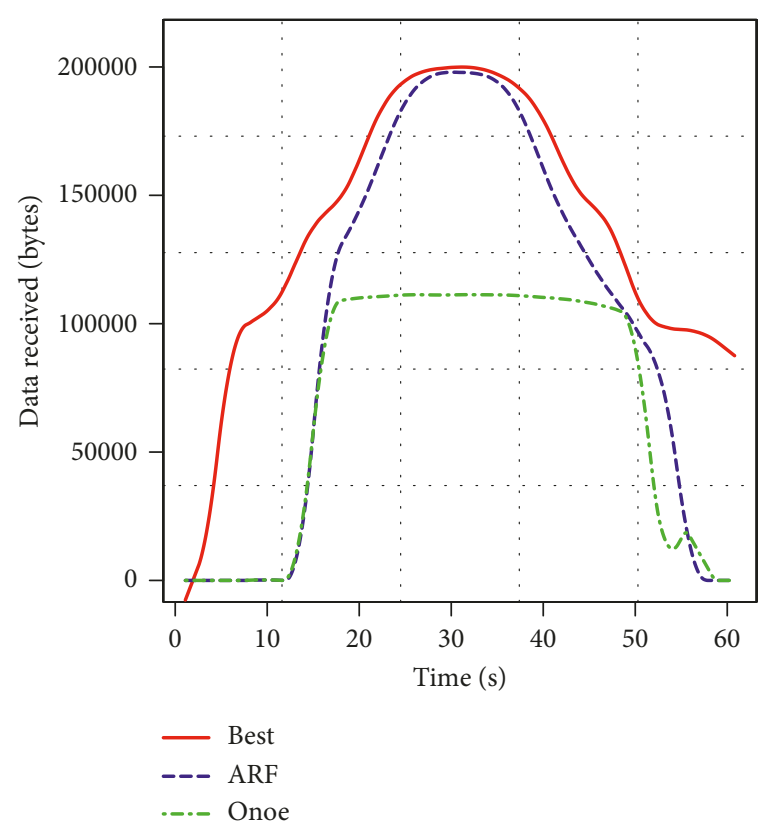

(b)

Figure 3: Communication performance of FTP and CBR for ARF and Onoe. (a) FTP. (b) CBR.

TABLE 2: Communication performance of FTP and CBR for ARF and Onoe.

\begin{tabular}{|c|c|c|c|c|c|c|}
\hline & \multicolumn{2}{|c|}{$10 \mathrm{~km} / \mathrm{h}$} & \multicolumn{2}{|c|}{$60 \mathrm{~km} / \mathrm{h}$} & \multicolumn{2}{|c|}{$100 \mathrm{~km} / \mathrm{h}$} \\
\hline & ARF & Onoe & ARF & Onoe & $\mathrm{ARF}$ & Onoe \\
\hline \multicolumn{7}{|l|}{ FTP } \\
\hline Best data received (MB) & \multicolumn{2}{|c|}{222} & \multicolumn{2}{|c|}{37.2} & \multicolumn{2}{|c|}{21.9} \\
\hline Data received $(\mathrm{MB})$ & 166 & 133 & 24.6 & 19.9 & 12.7 & 10.5 \\
\hline Room for improvement (\%) & 25.3 & 40.0 & 33.9 & 46.5 & 42.0 & 52.0 \\
\hline \multicolumn{7}{|l|}{ CBR } \\
\hline Best data received (MB) & \multicolumn{2}{|c|}{503} & \multicolumn{2}{|c|}{78.8} & \multicolumn{2}{|c|}{45.9} \\
\hline Data received $(\mathrm{MB})$ & 374 & 259 & 57.2 & 38.9 & 31.2 & 21.7 \\
\hline Room for improvement (\%) & 25.6 & 48.5 & 27.4 & 50.6 & 32.0 & 52.7 \\
\hline
\end{tabular}

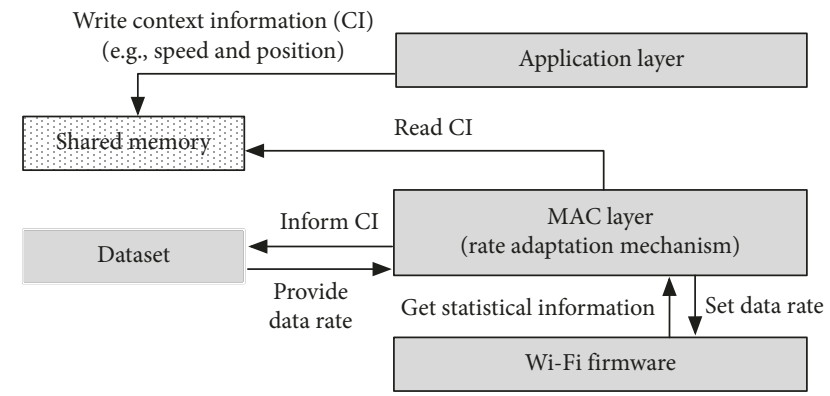

Figure 4: Design overview of the proposed method.

quality, the proposed method also employs data rate selection based on measurement information.

4.2. Dataset for Selecting a Data Rate. As mentioned in Section 2, the existing methods have several drawbacks for adapting a data rate for various situations. First, they raise and lower the data rate stepwise based on measurement information. In a movement environment where channel quality is fluctuating continuously and abruptly, as it is impossible to estimate channel quality without any delay, the estimated result may be obsolete. Hence, even if there were an opportunity to use a higher data rate, they might continue to use a lower data rate. Second, at the beginning of communication, since the channel quality is unknown, it takes time to evaluate the present channel quality and select a data rate. Besides, the data rate used begins with the lowest data rate. Thus, in $\mathrm{Wi}$-Fi communication involving shared media, since the use of excessively low data rate causes the communication performance of the whole $\mathrm{Wi}-\mathrm{Fi}$ system to degrade, an appropriate data rate must be selected promptly.

We therefore propose a rate adaptation mechanism based on a dataset. As described above, the dataset is assumed to consist of three-tuple information of the vehicle's location, speed, and data rate, that is, a vehicle can obtain data rate information suitable for its location and speed from the dataset. Employing the dataset, the proposed method contributes to reducing the use of an excessively low data rate, because it can select a suitable data rate without any delay of measurement. However, if inappropriate information is 
provided, many frames may be frequently lost. To avoid such deterioration of communication performance, when a data rate selected is not suitable for the present channel quality, the proposed method switches to a data rate selection based on measurement. For this research, we employ a dataset that has collected and analyzed data beforehand.

4.3. Available Data Rate Trimming. Existing rate adaptation methods usually use all data rates, i.e., eight data rates for $802.11 \mathrm{p}$. On the other hand, the proposed method reduces the available data rates. Thus, in the case of $802.11 \mathrm{p}$, it employs only four data rates among eight data rates. Figure 5 shows the relationship between data received and data rate from an analysis of the results in Section 3. The figure plots data rate used for the best value in the CBR graph of Figure 2 and shows that data rates used are almost the top four data rates, i.e., $12,18,24$, and $27 \mathrm{Mbit} / \mathrm{s}$. Consequently, the proposed method employs only these four data rates.

Reducing the number of data rates leads to the following advantage. If all data rates are employed, it becomes more difficult to follow the present channel quality because of the time required to retransmit frames and the long occupancy of a channel when frame loss occurs in a low data rate. On the other hand, as shown in Figure 5, in the proposed method, as low data rates are not employed by trimming the number of available data rates, the opportunities for utilizing high data rates are increased, that is, the proposed method is expected to improve communication performance.

4.4. Inappropriate Data Rate Information from Dataset. The proposed method primarily provides suitable data rate information based on CI and a dataset. However, the data rate information does not always give an appropriate data rate due to various indefinite factors such as multipath fading and shadowing. Also, in the early phase of the system, the dataset itself may not be deployed at all locations.

The proposed method mainly utilizes data rate information suggested from the dataset to determine a suitable data rate. While employing this data rate, if a fixed number of consecutive frames are lost, the proposed method additionally prepares another data rate that is lower than the data rate provided by the dataset and then uses both of them alternately to transmit frames. This is because the method is designed to avoid unsuitable deterioration of the data rate due to burst lost frames. For instance, if a burst loss occurs during movement, the duration of the loss cannot be predicted. Also, if the data rate is immediately downgraded in reaction to the loss, this will cause unnecessary channel occupation due to the usage of a lower data rate, which might lead to the degradation of communication performance.

To alleviate the effect of such degradation, the proposed method uses two data rates and also brings a flexible data

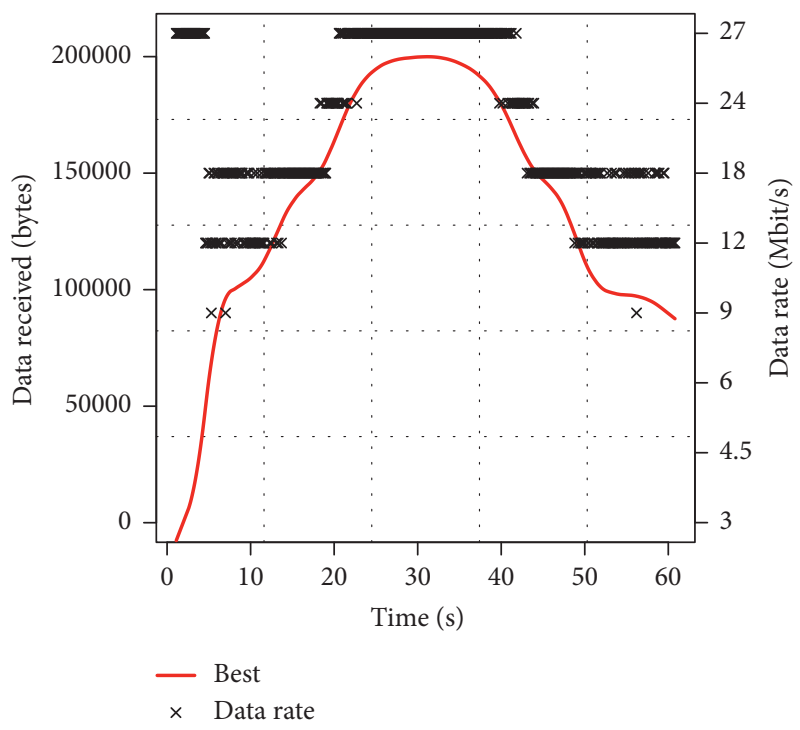

FIgURE 5: Relationship between data received and data rate in the best value result.

rate selection by utilizing the measurement result of channel quality. While it is employing two data rates, the proposed method records information regarding the success and failure of frames transmitted from each data rate and the elapsed time for transmission. This information is kept as an exponential moving average.

In communication for a certain period using two data rates, if only the higher data rate of two data rates experiences loss, the proposed method switches to using only the lower data rate for the communication. Besides, if both data rates experience loss, the data rates in use are downgraded to the next lower data rate. On the other hand, from a measurement result obtained during a certain period, if the number of frames lost at both data rates is lower than a predetermined threshold, the loss of consecutive frames is determined to be an accidental event, and the data rate in use is then switched to the higher data rate. Algorithm 1 illustrates the flexible data rate selection method described above.

4.5. Data Rate Selection with a Dataset and Measurement. A dataset may not always be able to provide adequate data rate information, and also the dataset may not be distributed in the early stage. To adequately work in such situations, the proposed method is equipped with a data rate selection based on measurement of channel quality, in addition to the dataset.

The proposed method employs statistical information about throughput as a measurement metric for channel quality. The throughput is calculated as follows:

$$
\text { throughput }=\frac{\text { successfully transmitted bytes (TxBytes) }}{\text { occupation period for a channel by a data rate (airtime) }},
$$


(1) use data rate based on dataset

(2) IF a fixed number of consecutive frames are lost THEN

(3) use two data rates (the current data rate and a data rate lower than the current data rate)

(4) WHILE using two data rates

(5) measure lost frames for each data rate

(6) IF both data rates have no lost frames THEN

(7) use the higher data rate

(8) ELSE IF only the higher data rate has lost frames THEN

(9) use the lower data rate

(10) ELSE IF both data rates have lost more frames than a predetermined threshold THEN

(11) use a third data rate lower than the lower data rate

(12) ELSE IF both data rates have lost fewer frames than a predetermined threshold THEN

(13) use the higher data rate

(14) ENDIF

(15) ENDWHILE

(16) ENDIF

Algorithm 1: Flexible data rate selection.

where TxBytes means the number of bytes for successfully transmitted frames within a certain period at a given data rate, while airtime denotes an accumulation time from sending a data frame to receiving an ACK frame for the data frame sent.

The proposed method keeps statistical throughput information for each data rate separately. After a certain period for a measurement, it selects the data rate that has the largest throughput as a data rate based on measurement information. Then, by comparing with a data rate based on the dataset and the measurement, if they both provide the same data rate or if the data rate based on the measurement is lower than that of the dataset, the proposed method switches back to the control based on the dataset.

\section{Performance Evaluation}

This section provides communication performance comparisons for the proposed method and the existing methods ARF and Onoe. Sections 5.1 and 5.2 show results of communication performance of both FTP and CBR applications for 10,60 , and $100 \mathrm{~km} / \mathrm{h}$ in single- and multiple-vehicle cases, respectively.

5.1. Result for a Single Vehicle. The evaluation employs the same simulation model, with the parameters explained in Section 3. In the simulation experiments, in addition to ARF and Onoe, as the proposed method we employ two rate adaptation mechanisms: rate adaptation with dataset and measurement (RA-DM) and rate adaptation with dataset (RAD). RA-DM utilizes both dataset and measurement functions, while RAD controls data selection by dataset information.

Table 3 shows the simulation results for the four methods. The evaluation shows room for improvement as the comparison metric; this means that a smaller value is approaching the best value. From the results, in the case of a single vehicle, we can see that RA-D shows the worst performance for FTP communication among the four, while it gives the best performance for CBR communication. This is because, since RA-D selects a data rate based only on the information from the dataset, it does not
TABLE 3: Room for improvement in a single-vehicle case.

\begin{tabular}{lcccccc}
\hline Speed & \multicolumn{2}{c}{$10 \mathrm{~km} / \mathrm{h}$} & \multicolumn{2}{c}{$60 \mathrm{~km} / \mathrm{h}$} & \multicolumn{2}{c}{$100 \mathrm{~km} / \mathrm{h}$} \\
Application & FTP & CBR & FTP & CBR & FTP & CBR \\
\hline ARF (\%) & 25.5 & 25.7 & 34.0 & 27.5 & 41.7 & 31.9 \\
Onoe (\%) & 40.0 & 48.5 & 46.4 & 50.7 & 51.8 & 52.6 \\
RA-D (\%) & 77.2 & 25.2 & 79.9 & 26.5 & 81.4 & 29.9 \\
RA-DM (\%) & 32.1 & 31.4 & 39.1 & 33.8 & 46.2 & 43.4 \\
\hline
\end{tabular}

change the data rate in use to another data rate even if frame loss occurs. Consequently, consecutive frame loss leads to packet loss and delay, and then FTP performance degrades due to the retransmission control of transmission control protocol (TCP). On the other hand, in CBR communication, since user datagram protocol (UDP) employed for CBR continues to send packets without retransmission control even if packet loss occurs, the CBR performance is improved to become the best of the four methods.

In the result of RA-DM, the communication performances for both FTP and CBR are better than those of Onoe, but somewhat worse than those of ARF. The result indicates that RA-DM can follow the changes in channel quality more effectively for both FTP and CBR applications than Onoe and FTP of RA-D.

5.2. Result for Multiple Vehicles. The previous result showed that the performance of ARF is somewhat higher than RADM. However, in environments where multiple vehicles exist, ARF may not be able to sustain that excellent performance, because an increase in the number of vehicles prolongs the waiting time to send frames due to the characteristics of shared media. To evaluate the impact of such an effect, this section evaluates simulation models involving two and ten vehicles.

The simulation model and parameters are the same as those for the case of a single vehicle. From the initial evaluation, all vehicles are assumed to be driving at the same location, time, and speed. 
TABLE 4: Improvement ratio of RA-DM to ARF, Onoe, and RA-D in two-vehicles case.

\begin{tabular}{lcccccr}
\hline Speed & \multicolumn{2}{c}{$10 \mathrm{~km} / \mathrm{h}$} & \multicolumn{2}{c}{$60 \mathrm{~km} / \mathrm{h}$} & & $100 \mathrm{~km} / \mathrm{h}$ \\
Application & FTP & CBR & FTP & CBR & FTP & CBR \\
\hline RA-DM/ARF & 1.52 & 1.00 & 1.57 & 0.95 & 1.43 & 0.92 \\
RA-DM/Onoe & 1.11 & 1.42 & 1.15 & 1.27 & 1.06 & 1.26 \\
RA-DM/RA-D & 2.94 & 1.10 & 2.92 & 1.17 & 2.99 & 1.29 \\
\hline
\end{tabular}

TABLE 5: Improvement ratio of RA-DM to ARF, Onoe, and RA-D in ten-vehicles case.

\begin{tabular}{lcccrrr}
\hline Speed & \multicolumn{2}{c}{$10 \mathrm{~km} / \mathrm{h}$} & & $60 \mathrm{~km} / \mathrm{h}$ & & $100 \mathrm{~km} / \mathrm{h}$ \\
Application & FTP & CBR & FTP & CBR & FTP & CBR \\
\hline RA-DM/ARF & 2.33 & 3.87 & 2.52 & 3.94 & 2.28 & 3.41 \\
RA-DM/Onoe & 1.06 & 3.12 & 1.00 & 1.58 & 0.98 \\
RA-DM/RA-D & 2.67 & 0.91 & 3.26 & 0.85 & 2.90 & 0.77 \\
\hline
\end{tabular}

Tables 4 and 5 show the improvement ratio for the four methods in the two- and ten-vehicles cases, respectively.
Here, as the comparison metric, we define the improvement ratio calculated as follows:

$$
\text { the improvement ratio of RA-DM }=\frac{\text { the number of communication bytes on RA-DM }}{\text { the number of communication bytes on }\{\text { ARF } \mid \text { Onoe } \mid \text { RA-D }\}} \text {. }
$$

The results demonstrate that the communication performances of RA-DM are overall improved in both cases. Moreover, the result for ARF is degrading with the increase of vehicles, while that for Onoe is improving. This is because, as the waiting time to send frames is prolonged as the number of vehicles increases, ARF is late in catching up with the changes in channel quality. The reason why the result for Onoe is improving is not that Onoe follows changes in channel quality but that the number of switches in data rates is small. That is, as ARF and Onoe necessarily transmit frames in order to change the data rate that starts with the lowest data rate, it is late to follow the changes.

On the other hand, the proposed method provides the following three features in order to improve communication performance. First, it employs higher data rates by trimming regular data rates, and it can then begin with an appropriate data rate based on a database. Lastly, it prepares the data rate selection based on measurement as a countermeasure against inappropriate data rate information from the dataset.

In the one-vehicle simulation result, RA-D has the best performance for the CBR traffic model, and it also has the worst performance for the FTP traffic model. However, to adapt to TCP and UDP traffics, a rate adaptation mechanism needs to improve the communication performance for both. On the other hand, the improvement of RA-DM is lower than that of the counter-based mechanism (ARF) but higher than that of the sample-based mechanism (Onoe).

On the other hand, in the case where multiple vehicles exist, RA-DM generally has a better communication performance than counter-based and sample-based mechanisms. On the other hand, in the case of CBR for ten vehicles, RA-D outperforms RA-DM if we can know the number of vehicles, but it is difficult to estimate it. Therefore, the proposed method (RA-DM) provides superior communication performance in situations involving more than one vehicle.

\section{Conclusion}

In this study, to improve communication performance between a connected vehicle and RSU using Wi-Fi during movement in a V2I environment, we proposed a rate adaptation mechanism introducing the following new two approaches: available data rate trimming and data rate information provision. In the available data rate timing, the proposed method selected four data rates among the standard eight data rates to avoid usages of excessively low data rates. Also, it utilizes data rate information provided from a dataset in order to select a suitable data rate without any delay of measurement. It does not, however, work well in every situation because of various indefinite factors such as multipath fading and shadowing. To compensate for the drawbacks of the dataset, the proposed method then also employed a data rate selection based on measurement. In the simulation experiments, we investigated the FTP and CBR communication performance in single- and multiplevehicle cases. In a single-vehicle case, RA-D shows the worst performance for FTP communication among the four methods, while it gives the best performance for CBR communication. On the other hand, RA-DM provides better communication performance for both FTP and CBR next to ARF. In two- and ten-vehicles cases, communication performance of ARF is getting worse with increasing the number of vehicles, while RA-DM overall provides superior communication performance in 
situations involving more than one vehicle, in comparison with other methods.

\section{Data Availability}

The data used to support the findings of this study are available from the corresponding author upon request.

\section{Conflicts of Interest}

The authors declare that they have no conflicts of interest.

\section{Acknowledgments}

This work was supported by JSPS KAKENHI (Grant number: 18H03232).

\section{References}

[1] E. Ndashimye, S. K. Ray, N. I. Sarkar, and J. A. Gutiérrez, "Vehicle-to-infrastructure communication over multi-tier heterogeneous networks: a survey," Computer Networks, vol. 112, pp. 144-166, 2017.

[2] R. Coppola and M. Morisio, "Connected car: technologies, issues, future trends," ACM Computing Surveys, vol. 49, no. 3, pp. 1-36, 2016.

[3] K. Kai, W. Cong, and L. Tao, "Fog computing for vehicular Ad-hoc networks: paradigms, scenarios, and issues," Journal of China Universities of Posts and Telecommunications, vol. 23, no. 2, pp. 56-96, 2016.

[4] N. Cheng, N. Lu, N. Zhang, X. Shen, and J. W. Mark, "Vehicular WiFi offloading: challenges and solutions," Vehicular Communications, vol. 1, no. 1, pp. 13-21, 2014.

[5] IEEE Computer Society, Part 11: Wireless LAN Medium Access Control (MAC) and Physical Layer (PHY) Specifications, IEEE Standards Association, IEEE Computer Society, Washington, DC, USA, 2012.

[6] E. Ahmed and H. Gharavi, "Cooperative vehicular networking: a survey," IEEE Transactions on Intelligent Transportation Systems, vol. 19, no. 3, pp. 996-1014, 2018.

[7] A. Kamerman and L. Monteban, "WaveLAN ${ }^{\circledR}$-II: a highperformance wireless LAN for the unlicensed band," Bell Labs Technical Journal, vol. 2, no. 3, pp. 118-133, 2002.

[8] M. Lacage, M. H. Manshaei, and T. Turletti, "IEEE 802.11 rate adaptation: a practical approach," in Proceedings of the 7th ACM International Symposium on Modeling, Analysis and Simulation of Wireless and Mobile Systems, pp. 126-134, Venice, Italy, October 2004.

[9] Q. Xia, J. Pu, and M. Hamdi, "Model-tree-based rate adaptation scheme for vehicular networks," in Proceedings of the 2009 IEEE International Conference on Communications, pp. 1-5, Dresden, Germany, June 2009.

[10] C. Liu, S. Liu, and M. Hamdi, "GeRA: generic rate adaptation for vehicular networks," in Proceedings of the 2012 IEEE International Conference on Communications (ICC), pp. 5311-5315, Ottawa, ON, Canada, June 2012.

[11] P. Shankar, T. Nadeem, J. Rosca, and L. Iftode, "CARS: context-aware rate selection for vehicular networks," in Proceedings of the 2008 IEEE International Conference on Network Protocols (ICNP), pp. 1-12, Orlando, FL, USA, October 2008.

[12] J. He, H. Liu, P. Cui et al., "Design and experimental evaluation of context-aware link-level adaptation," in Proceedings of the 2012 Proceedings IEEE INFOCOM, pp. 2726-2730, Orlando, FL, USA, March 2012.

[13] Y. Song, X. Zhu, Y. Fang, and H. Zhang, "Threshold optimization for rate adaptation algorithms in IEEE 802.11 WLANs," IEEE Transactions on Wireless Communications, vol. 9, no. 1, pp. 318-327, 2010.

[14] S. H. Y. Wong, H. Yang, S. Lu, and V. Bharghavan, "Robust rate adaptation for 802.11 wireless networks," in Proceedings of the 12th Annual International Conference on Mobile Computing and Networking (MobiCom '06), pp. 146-157, Los Angeles, CA, USA, September 2006.

[15] S. Biaz and S. Wu, "Rate adaptation algorithms for IEEE 802.11 networks: a survey and comparison," in Proceedings of the 2008 IEEE Symposium on Computers and Communications, pp. 130-136, Marrakech, Morocco, July 2008.

[16] J. C. Bicket, Bit-Rate Selection in Wireless Networks, Massachusetts Institute of Technology, Cambridge, MA, USA, 2005.

[17] Linux Wireless, September 2018, https://wireless.wiki.kernel. org/en/developers/documentation/mac80211/ratecontrol/ minstrel.

[18] D. Xia, J. Hart, and Q. Fu, "Evaluation of the minstrel rate adaptation algorithm in IEEE 802.11g WLANs," in Proceedings of the 2013 IEEE International Conference on Communications (ICC), pp. 2223-2228, Budapest, Hungary, June 2013.

[19] J. Zhang, K. Tan, J. Zhao, H. Wu, and Y. Zhang, "A practical SNR-guided rate adaptation," in Proceedings of the IEEE INFOCOM 2008-The 27th Conference on Computer Communications, pp. 2083-2091, Phoenix, AZ, USA, April 2008.

[20] A. Vlavianos, L. K. Law, I. Broustis, S. V. Krishnamurthy, and M. Faloutsos, "Assessing link quality in IEEE 802.11 wireless networks: which is the right metric?," in Proceedings of the 2008 IEEE 19th International Symposium on Personal, Indoor and Mobile Radio Communications, pp. 1-6, Cannes, France, September 2008.

[21] G. Judd, X. Wang, and P. Steenkiste, "Efficient channel-aware rate adaptation in dynamic environments," in Proceedings of the 6th International Conference on Mobile Systems, Applications, and Services-MobiSys '08, pp. 118-131, Breckenridge, CO, USA, June 2008.

[22] J. Xiong, C. Chen, X. Guan, C. Hua, and LRRA, "Locationrelated rate adaptation algorithm in IEEE 802.11p for DSRC technology in VANET," in Proceedings of the 2016 IEEE 84th Vehicular Technology Conference (VTC-Fall), pp. 1-5, Montréal, Canada, September 2016.

[23] O. Puñal, H. Zhang, and J. Gross, "RFRA: random forests rate adaptation for vehicular networks," in Proceedings of the 2013 IEEE 14th International Symposium on "A World of Wireless, Mobile and Multimedia Networks" (WoWMoM), pp. 1-10, Madrid, Spain, June 2013.

[24] A. Zekri and W. Jia, "Performance evaluation of rate adaptation algorithms in IEEE802.11p heterogeneous vehicular networks," in Proceedings of the 2018 IEEE 15th International Conference on Mobile Ad Hoc and Sensor Systems, MASS, vol. 2018, pp. 107-115, Chengdu, China, October 2018.

[25] ETSI, Intelligent Transport Systems (ITS); Vehicular Communications; Basic Set of Applications; Local Dynamic Map (LSM), ETSI EN 302895 V1.0.0 (2014-01), ETSI, Sophia Antipolis, France, 2014. 


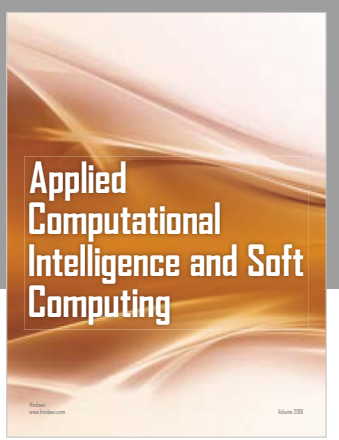

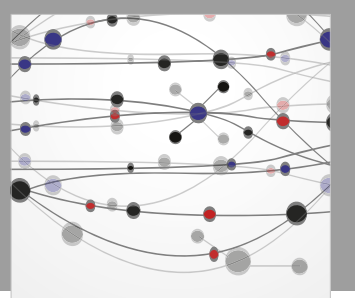

The Scientific World Journal
Submit your manuscripts at

Computing
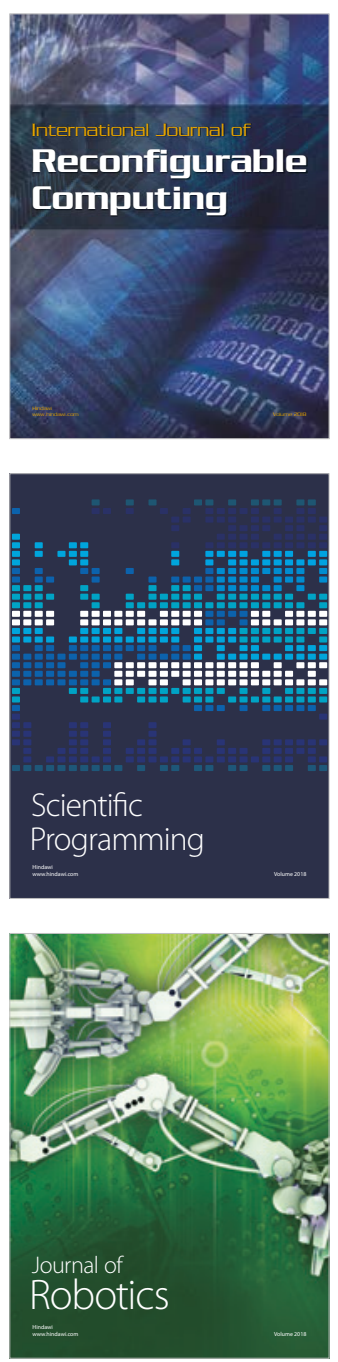

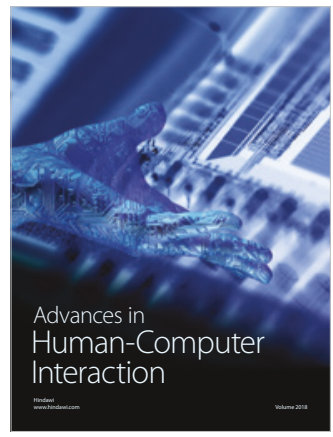

Human-Compute

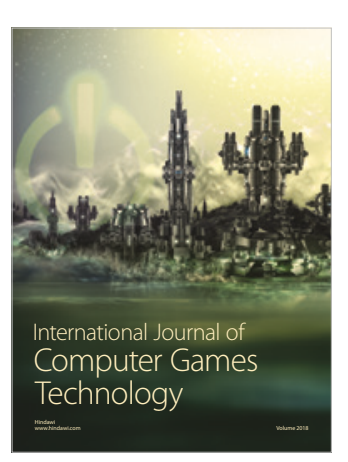

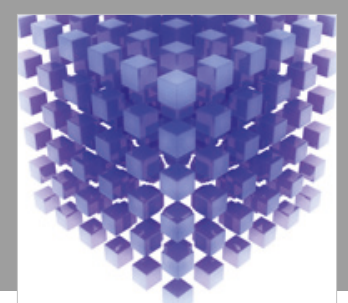

Mathematical Problems in Engineering

\section{Engincering}
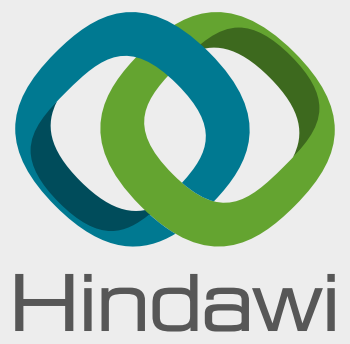

www.hindawi.com
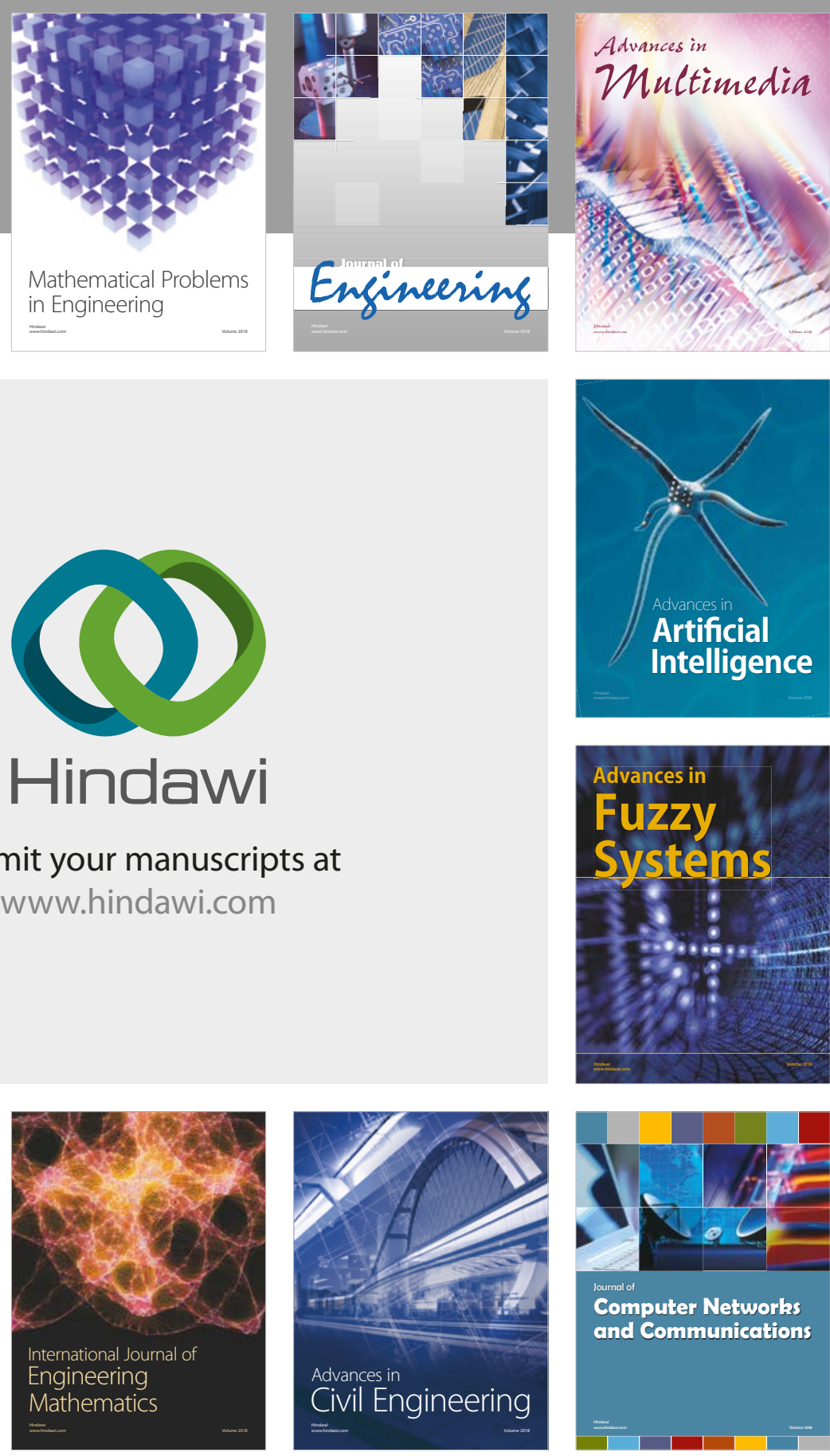

Computer Networks and Communications

Multimedia
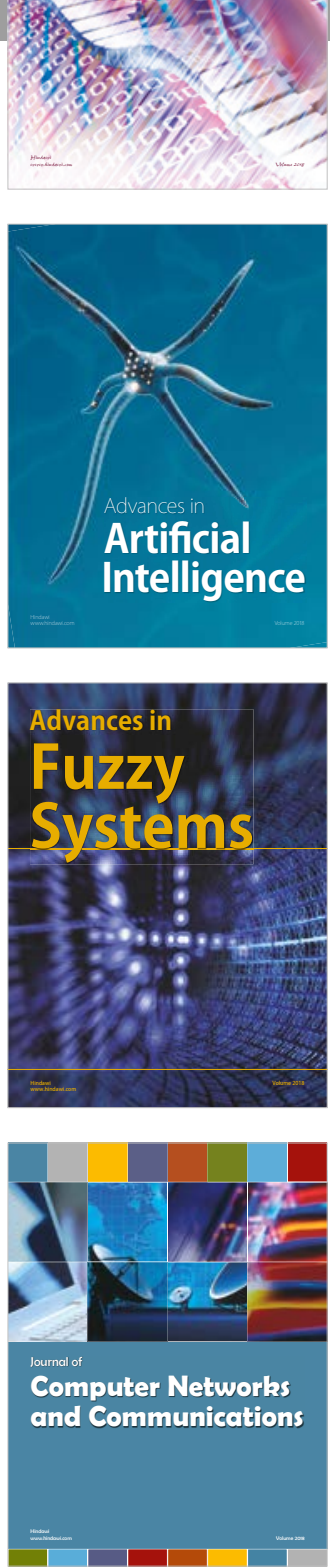

Advances in

Modelling \&

Simulation

in Engineering

interaction

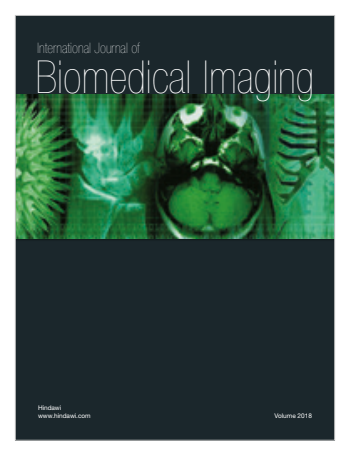

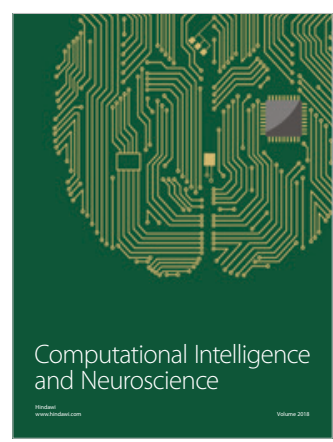

